\title{
肾病综合征出血热病毒形态发育中的 一些特殊形态
}

\author{
洪 涛 周新仪 杭长寿
}

（中国预防医学中心病毒学研究所，北京）

肾病综合征出血热 (HFRS) 虽有多年的流行历史, 然而其病原学研究却刚刚突破 ${ }^{(1-3)}$ 。近 来我国首先在感染细胞内发现了 HFRS 病毒的形态, 论证它为一组新的布尼雅病毒 ${ }^{[4-6]}$. 在 我们以前的报告中还证明我国不同地区及不同类型的 HFRS 病毒具有相同的形态 ${ }^{[7]}$. 本 文 报道 HFRS 病毒在细胞内发育中所显示的一些特有的形态学特征.

\section{一、材料与方法}

1. 病毒-细胞系统

（1）病毒: $A_{9}, A_{5}$ 株均从流行性出血热疫区野鼠分离得来; $R_{12}, R_{27}, R_{1}$ 株均从流行 性出血热疫区家鼠分离得来.

(2) 细胞: Vero- $\mathrm{E}_{6}$.

2. 感染方法: 将病毒原液 $0.5-1 \mathrm{ml}$ 接种于小方瓶单层细胞上, $37^{\circ} \mathrm{C}$ 吸附 2 小时, Hank's 液洗 3 次,换 Eagle 维持液, $37^{\circ} \mathrm{C}$ 培养,逐日观察, 2-3 天换液 1 次,感染后 10-15 天收获做 常规电镜固定, 包埋切片和电镜观察. 本文实验使用 Sorvall MT5000 和 LKB Nova 超薄切 片机，用钻石刀切片，电镜为 JEM-100B 和 JEM-1000x。

\section{二、结 果}

总的说来, HFRS 病毒具有布尼雅病毒的基本形态特征, 由一个单位膜包括着颗粒丝状 的病毒浆, 病毒颗粒的大小范围跨度甚大, 我们测量的最小者 $78 \mathrm{~nm}$, 最大者 $201 \mathrm{~nm}$, 平均大小 为 $122 \mathrm{~nm}$ (图 1、2). 有关该病毒的大小、形态已在先前论文中报告过,不再鳌述了. 现将该病 毒在发育和释放过程中出现的一些特殊的形态描述如下:

1. 两种类型的包含体在病毒感染的细胞内总是可以见到两种大小相似而结构不同的包含 体.

（1）颗粒状包含体：此种包含体一般较小，而形状较圆，由无数微小的电子致密颗粒组 成, 颗粒之间往往由细丝串联成短链或颗粒网状 (图 3a), 包含体边缘没有明显的界限和包膜, 与胞浆基质混为一体. 然而, 包含体周围总是有较多的聚合核蛋白体的集结, 并往往与扩大 增殖的粗面内质网靠近或直接相连. 值得特别指出的是在这种包含体的附近往 往可以发现 HFRS 病毒颗粒, 有的颗粒在包含体附近的震腔内, 有的与包含体直接相连(图 3b).

本本1984年5月7日收到. 
（2）颗粒丝状包含体: 这种包含体一般较大而形状不规则,呈三角形，棱形或柏圆形，常 常敖近细胞核 (图 4). 包含体的颗粒性结构成份与以上颗粒包含体无明显差别, 而在颗粒的 基质上出现成束的电子致密的微管状结构, 微管直径约 $76 \AA$, 微管往往分布在包含体的边缘 部或一端, 呈规则排列, 其横断面皇中空的晶格状(图 $3 \mathrm{a}$ 箭头所示), 在此种包含体内有时可见 到个别较大而不典型的病毒样颗粒或空泡状结构.

在包含体的附近的胞浆基质中往往可见到散在的微丝和成串的聚合核蛋白体.

\section{2. 细胞表面的均质性结构层}

在感染的细胞表面, 尤其是在有病毒颗粒存在的细胞表面, 常常见到由均质性物质构成厚 度 $42 \mathrm{~nm}$ 的一层电子致密结构, 它既不是病毒的本身, 又不是细胞质膜, 却很象由细胞分䎵的一 层粘液性物质夏盖在细胞表面, 由于它只能在感染的细胞上见到而且总是与病毒颗粒相伴随, 经特异的酶标兔度电镜技术证明为 HFRS 相关抗原,显然它与病毒的发育有关(图 4b).

\section{3. 病毒相随颗粒}

在 HFRS 病毒发育过程中出现的一个非常独特的现象, 即病毒颗粒相随颗柆, 这种颗粒 密度很深, 直径约 $32 \mathrm{~nm}$, 形态与核蛋白颗粒相似. 值得特别指出的是, 这种颗粒与病毒颗粒 之间的密切关系, 有时它们与不完整的病毒颗粒混为一体 (图 4a 箭头所示), 有时直接联入病 毒颗粒的单位膜里或附着在病毒颗粒的表面. 然而, 并非所有的病毒颗粒都伴有此种颗柆, 目 前还找不出它们出现的规律. 在免疫酶标染色切片上, 由于它们着色很深而难以与免疫沉腚 物相鉴别 (图 2)。

\section{4. 异形颗粒}

在大量的超薄切片观察中除了通常的病毒颗粒之外,还发现了一些异常颗粒,其中包括:

（1）榊蚪状颗粒: 这种颗粒形似蝌蚪，有一个由双层单位膜构成的粗尾部和与通常成熟 的 HFRS 病毒颗柆相同的头部, 其尾部末端与细胞浆内质网壁相连, 很象噬菌体颗粒或破土 而出的藤姑 (图 4d), 这种颗粒有时也可在细胞外见到.

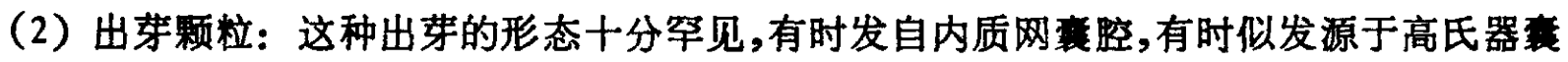
泡中(图 4c). 发芽的颗粒除了底部与衰泡相连外, 其它结构与细胞内外的 HFRS 病毒颗粒无 明显差别,单位膜和病毒浆均清晰可见.

（3）管状颗粒：在感染细胞的胞浆空泡中,有时可出现管状的颗粒（图 4e), 管壁系由挺 直的单位膜组成, 膜的厚度与通常成熟颗粒者相当, 其横断面直径约 $70 \mathrm{~nm}$, 呈环状, 中间看不

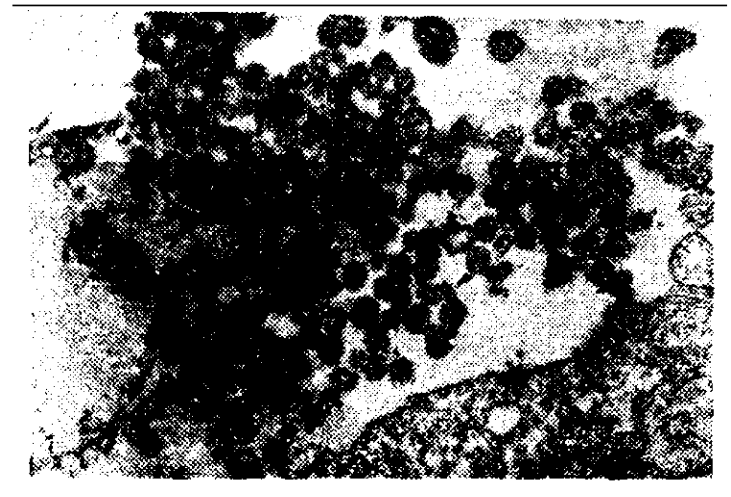

图 1 细胞外的 HFRS 病毒颗粒 图中可雷出 HFRS 病颗粒大小的差别, $\triangle$ 示病繁相随颗粒

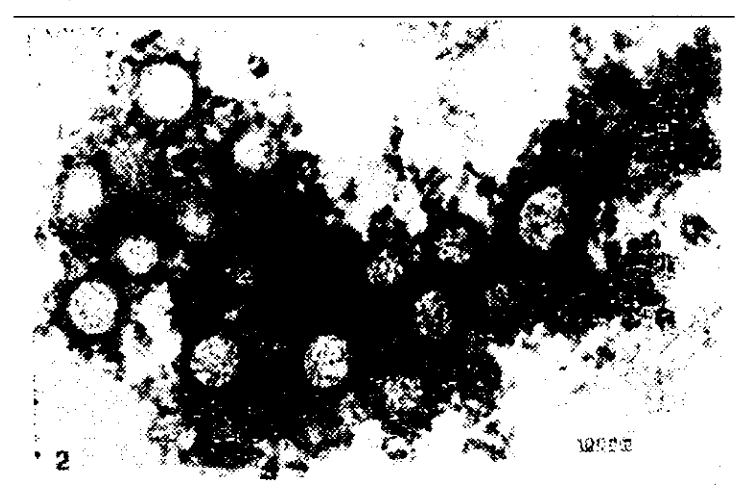

图 2 HFRS 病的免政标电镜照片

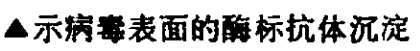




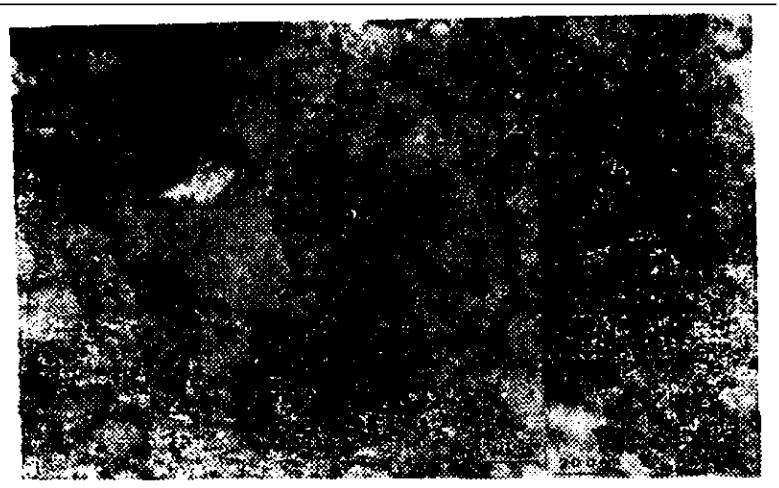

图 3 示 HFRS 病毒细胞浆内颗粒包含体 (IB) A示病颗粒及其相随颗粒; $\uparrow \uparrow$ 示增殖的聚合核勇白 颗粒构成特殊的排列; 32 为细胞浆内営看到的另一 种微丝包含体; 3b 示包含体周围的 HFRS 病哑颗柆

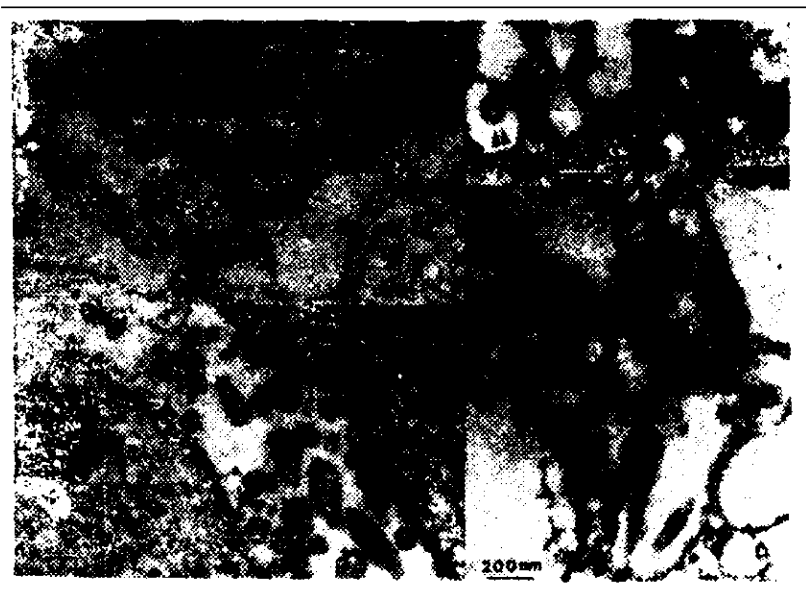

图 4

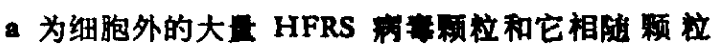
(A); b 二个细胸表面均质结约层(A)和 HFRS

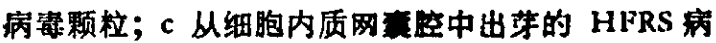

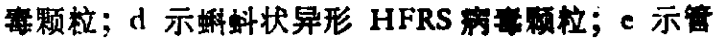
状颗粒及其横断面( $(\Delta)$

到成熟颗粒中所见的那种特有颗粒丝状病毒浆 (图 4e 箭头所示), 相似的空心环状颗粒也时常 可在细胞外颗粒中见到, 其大小和内部充实的程度有很大差别,有的完全空譪, 有的在其内部 可见少量颗粒丝状物质.

\section{三、讨 论}

在我们以前的论文中，根据 HFRS 病毒总的形态学特征,建议把它们归属于一组新的布 尼雅病毒. 进一步的研究说明 HFRS 病毒在形态发育中,确实具有突出的特征 ${ }^{[13]}$ ，其中包括 本文描述的包含体，感染细胞表面的均质性电子致密结构层和病毒相随颗粒等．这些特殊结 构以前既未在布尼雅病毒中发现, 也未见于其它病毒. 这很可能是因为:（i） HFRS 病毒确 实不同于已知的任何布尼雅病毒（ii）本文所描述的特殊结构尚末在其它布尼雅病毒中发现， 说明布尼雅病毒的形态学研究还不充分,有待深人.

Murphy 等曾在李夫特谷热病毒感染的鼠脑中发现过病毒基质结构, 然而他所描述的病毒 基质远不是本文中所描述的包含体, 此外也从未把包含体结构作为布尼雅病毒发育的重要特 征 ${ }^{[8]}$, 而 Ellis 等也曾在李夫特谷热病毒感染的细胞核里发现过包含体, 显然也与本文所见不 同 ${ }^{[9]}$. 我们的实验反复证明包含体在 HFRS 形态发育中起重要作用, 它形态特殊而数量很 多,几乎可以在 HFRS 感染的所有细胞内见到, 而更为重要的是病毒颗粒似可直接从包含体

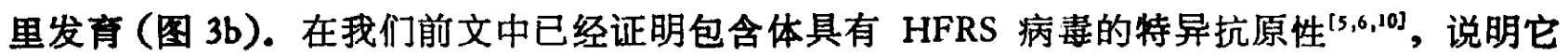
参与病毒的增殖. 本文描述了两种结构不同的包含体, 目前尚难肯定它们之间的关系, 鉴于只 是在颗粒性包含体附近见到了 HFRS 病毒颗粒,我们权且推测颗粒状包含体可能是颗粒丝状 包含体的进一步发展.

形态学和形态发生学是借以进行病毒分类的重要标准, 已知的各种病毒毫无例外都各有 自己固有的形态和形态发生学特征. 那么,如何判断 HFRS 的归属呢? 这组病毒总体形态与 布尼雅病毒相似,然而病毒颗粒远较其它布尼雅病毒大, 发育中包含体起到明显的作用, 此外 还有其它一些独有的特征, 如细胞表面的均质性结构物质, 病毒颗粒的相随颗粒等, 这些特有 
的形态学特征也许可以作为向导,最终把 HFRS 病毒作为布尼雅病毒中的独立的一属病琵或 作为它的一个亚科来考虑. 就病毒颗粒本身的形态看也与文献中已报道的布尼雅病毒有较大 的差别, 该病毒的负染电镜形态似也与一般布尼雅病毒颇不相同. 在我们先前的论文中曾经 指出, HFRS 病毒除了体积较大形态变异较多等特征之外, 病毒浆里含有比较粗大的颗粒丝 状结构,看上去与沙粒病毒颇有些相似 ${ }^{[12]}$. 反复地观察结果可以进一步认定 HFRS 病毒从形 态学上看是介于布尼雅病毒和沙粒病毒之间但更接近于布尼雅科的一组病毒, 深入的形态发 生学研究正在进行中.

\section{* * 文 新}

[ 1 ] Lee, H. W. et al., J. Infect Dis., 1978, 137-298.

[ 2 ] French, G. R. et al., Lancet, 1981, 1: 1070.

[3] 宋干等, 中国医学科学院学报, 4(1982), 2:73.

[4] Hung Tao et al., Lancet, 1983, 1: 589.

[ 5] 共涛等,中华微生物学免度学杂志, 3(1983), 2: 69 .

[ 6 ] Hung Tao et al., Archives of Virol, 78 (1983), 137-144.

[7] 等, 中国医学科学院学报, 5(1983), 5: 356-360.

[ 8 ] Murphy, F. A, et al., Intervinology, 1973, 1: 297-316.

[ 9 ] Ellis, D. S. et al., J. Gen. Virol., 40(1979), 329-339.

[10] Nocormik, J. B. et al., Lencet, 1982, 1: 765.

[11] Smith, J. F. et al., Virology, 121 (1982), 61-81.

[12] Murphy, F. A. et al., Bull. World Health Organ., 52(1975), 409-419.

[13] Hung Tao et al, Intervirology, 23(1985), 97-108 